\title{
Deformable liposomes for skin therapy with human epidermal growth factor: The effect of liposomal surface charge
}

\author{
Selenia Ternullo ${ }^{a}$, Purusotam Basnet ${ }^{\text {b,c }}$, Ann Mari Holsætera ${ }^{a}$, Gøril Eide Flatena, Louis \\ de Weerd $^{\text {d,e }}$, Nataša Škalko-Basnet ${ }^{\mathrm{a}, *}$
}

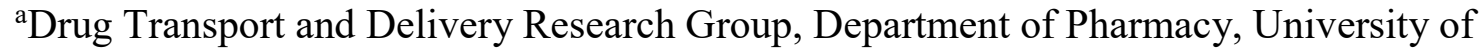
Tromsø The Arctic University of Norway, Universitetsveien 57, 9037 Tromsø, Norway ${ }^{\mathrm{b}}$ IVF Clinic, Department of Obstetrics and Gynecology, University Hospital of North Norway, Sykehusvegen 38, 9019 Tromsø, Norway

${ }^{\mathrm{c}}$ Women's Health and Perinatology Research Group, Department of Clinical Medicine, University of Tromsø The Arctic University of Norway, Universitetsveien 57, 9037 Tromsø, Norway

${ }^{\mathrm{d} D e p a r t m e n t ~ o f ~ P l a s t i c ~ a n d ~ R e c o n s t r u c t i v e ~ S u r g e r y, ~ U n i v e r s i t y ~ H o s p i t a l ~ o f ~ N o r t h ~ N o r w a y, ~}$ Sykehusvegen 38, 9019 Tromsø, Norway

${ }^{\text {e}}$ Research Group for Medical Imaging, Department of Clinical Medicine, University of Tromsø The Arctic University of Norway, Universitetsveien 57, 9037 Tromsø, Norway

*Corresponding author: Nataša Škalko-Basnet, Drug Transport and Delivery Research Group, Department of Pharmacy, University of Tromsø The Arctic University of Norway, Universitetsveien 57, 9037 Tromsø, Norway; Tel: +47-776-46640; Fax: +47-776-46151; Email: natasa.skalko-basnet@uit.no 


\begin{abstract}
The topical administration of exogenous human epidermal growth factor (hEGF) is a promising approach for improved chronic wound therapy. To develop therapeutically superior hEGF formulation, we prepared hEGF-containing neutral (NDLs), cationic (CDLs) and anionic (ADLs) deformable liposomes (DLs), respectively, since it is expected that the liposomal surface charge can affect both the liposomal physicochemical properties, their skin penetration potential and therapeutic efficacy of liposome-associated drug. All prepared liposomes were of similar size (300-350 nm) with high hEGF load ( 80\% entrapment efficacy). Among the studied DLs, ADLs were found to be most promising for sustained release of hEGF, as assessed in vitro using the polyamide membrane. Ex vivo studies revealed that all DLs were excellent systems for skin therapy with hEGF and no penetration of hEGF through the full thickness human skin was detected. ADLs provided a depot exhibiting the highest hEGF retention onto the human skin surface. ADLs also revealed enhanced mitogenic activities in human fibroblasts compared to both NDLs and CDLs after 48 hrs treatment. Moreover, hEGF-containing ADLs significantly enhanced mitogenic activity in fibroblast as compared to activity of hEGF solution (positive control). Similar trends were observed in human keratinocytes after $24 \mathrm{hrs}$ of treatment. We proved that the liposomal surface charge affects the therapeutic potential of hEGF-containing liposomes. hEGF-containing ADLs can be a promising nanosystem-based formulation for localized therapy of chronic wounds.
\end{abstract}

Key words: hEGF (human epidermal growth factor); deformable liposomes; liposome surface charge; human skin; chronic wound healing 
Abbreviations: ADLs, anionic deformable liposomes; CDLs, cationic deformable liposomes; DLs, deformable liposomes; ELISA, enzyme-linked immunosorbent assay; HaCaT, human immortalised keratinocytes; hEGF, human epidermal growth factor; HF, human foreskin fibroblasts; HLB, hydrophilic-lipophilic balance; IMDM, Iscove's modified Dulbecco's medium; NDLs, neutral deformable liposomes; PI, polydispersity index; PBS, phosphate buffer saline; SC, stratum corneum; SPC, soybean phosphatidylcholine. 


\section{Introduction}

The high prevalence of chronic wounds, their impact on patients' quality of life and the high healthcare costs call for an urgent need for improved chronic wound therapies (Hamdan et al., 2017). Moreover, the increasing proportion of high-risk population, such as diabetic, obese, and elderly people, further rises chronic wounds' incidence; it is estimated that up to $2 \%$ of the today's population will experience chronic wounds in their lifetime (Garcia-Orue, et al., 2017b; Ashtikar and Wacker, 2018). To enhance wound healing, novel strategies comprising agents to prevent/reduce wound infection, moisturize the wound, stimulate the healing mechanism and accelerate wound closing as well as scarring control are proposed (Kalashnikova et al., 2015). In the normal wound healing process, growth factors play a central role since they are involved in different phases of wound healing by promoting angiogenesis, stimulating the formation of granulation tissue and remodelling the reepithelisation (Jahromi et al., 2018). Chronic wounds fail to achieve the anatomic and functional integrity of the wounded area due to persistent inflammation, impaired angiogenesis, poor and delayed cellular proliferation and re-epithelialisation (Xue et al., 2018). The high levels of inflammatory cells are responsible for massive secretion of proteases in the wound bed. These enzymes degrade the growth factors, whose deficiency further delays the chronic wound healing (Saghazadeh et al., 2018). Human epidermal growth factor (hEGF) represents one of the main growth factors involved in the wound healing phases. Therefore, topical application of exogenous hEGF represents a promising approach for improving chronic wound therapy assuring faster and more effective wound healing (Garcia-Orue, et al., 2017b). However, there are several challenges related to the employment of hEGF in dermal therapy for treatment of chronic wounds. Major limitations related to local administration of hEGF are its low in vivo stability and rapid degradation by proteases present in the wound (Zeng and Harris, 2014). Those limitations would require frequent hEGF 
administrations to assure sufficiently high local concentrations for exerting its therapeutic effect (Gainza et al., 2015b). Subsequently, a systemic absorption and undesired side effects can occur.

Phospholipid-based nanosystems have shown to be promising dermal drug delivery systems for improved chronic wound therapy. Liposomes were employed as superior alternative to traditional drug delivery systems due to their excellent biocompatibility and biodegradability (Boateng and Catanzano, 2015). They can offer i) protection of the incorporated drug, ii) sustained and controlled drug release, and iii) drug accumulation onto/into the skin (Sala et al., 2018). All these functions exerted by liposomes might synergistically assure reduction in the frequencies of administration, high drug concentration at the skin site and avoidance of rapid absorption into the systemic circulation (Xue et al., 2018). Deformable liposomes (DLs) are designed as superior system in assuring effective dermal delivery of drugs (Sala et al., 2018). It has been demonstrated that the liquid-state of deformable liposome, together with their elastic membrane, allows them to better avoid aggregation and fusion under osmotic stress, which often occurs with conventional liposomes (Cevc, 2004). Moreover, conventional liposomes remain mainly confined in the upper epidermis layers where they can break due to lack in deformability thus loosening their content. This might cause uncontrolled dermal drug release. On the contrary, deformable liposomes can move into/through the skin layers by undergoing a stress-dependant adjustments thus resulting in more intact vesicles (Cevc, 2004) and limiting uncontrolled drug dermal delivery. The surface charge can play a role in modulating dermal drug delivery via liposomal systems (González-Rodríguez and Rabasco, 2011). When topically administered onto the skin, liposomes interact with the keratinocytes (Mota et al., 2017). It has been postulated that this interaction might be better promoted when liposomes are positively charged thus triggering electrostatic interactions with the negatively 
charged skin membrane (Jain et al., 2017). However, negatively charged liposomes have also proven their ability in promoting drug delivery through skin (Mota et al., 2017).

So far, different phospholipid-based nanosystems have been employed for dermal delivery of hEGF, such as conventional liposomes, solid lipid nanoparticles, nanostructured lipid carriers and nanofibers (Alemdaroğlu et al., 2008; Gainza et al., 2014; Garcia-Orue et al., 2017a; Jeon et al., 2012; Kaminski et al., 2016). To the best of our knowledge, deformable liposomes containing hEGF have only been investigated as positively charged nanosystems (Choi et al., 2017). Earlier, deformable liposomes have been shown effective (trans)dermal delivery systems for other proteins, such as gap junction proteins (Paul et al., 1995; Paul et al., 1998) and insulin (Cevc, 2003).

To explore the effect of liposomal charge on the mitogenic effect of hEGF, we prepared hEGF-containing neutral (NDLs), cationic (CDLs) and anionic (ADLs) deformable liposomes, respectively. All liposomes were characterised for in vitro hEGF release through polyamide membrane and ex vivo hEGF penetration through full thickness human skin. The toxicity of liposomes was evaluated in vitro by MTT assay. Finally, the mitogenic activity of hEGF-containing DLs was evaluated in vitro in human foreskin fibroblasts (HF) and human immortalised keratinocytes (HaCaT).

\section{Material and methods}

\subsection{Material}

Soybean phosphatidylcholine (Lipoid S100, SPC) was a generous gift from Lipoid GmbH (Ludwigshafen, Germany). hEGF, methanol CHROMASOLV ${ }^{\circledR}$, acetic acid, sodium phosphate dibasic dihydrate, potassium phosphate monobasic, sodium chloride, polysorbate 20, stearylamine and sodium deoxycholate were obtained from Sigma-Aldrich Chemie (Steinheim, Germany). 


\subsection{Preparation of liposomes}

Stock solution of hEGF $(1 \mathrm{mg} / \mathrm{mL})$ was prepared by dissolving hEGF $(500 \mu \mathrm{g})$ in $0.5 \mathrm{~mL}$ of $10 \mathrm{mM}$ acetic acid solution in MilliQ water, previously filtered through $0.20 \mu \mathrm{m}$ syringe filter (Bulk Acrodisc ${ }^{\circledR} 25$ mm Syringe Filter, Pall Life Sciences, East Hills, New York, USA). The stock solution was then diluted to the final concentration of $20 \mu \mathrm{g} / \mathrm{mL}$ with phosphate buffer saline (PBS) (pH 7.4, $2.98 \mathrm{~g} / \mathrm{L} \mathrm{Na}_{2} \mathrm{HPO}_{4} \times \mathrm{x}_{2} \mathrm{O}, 0.19 \mathrm{~g} / \mathrm{L} \mathrm{KH}_{2} \mathrm{PO}_{4}$ and $\left.8 \mathrm{~g} / \mathrm{L} \mathrm{NaCl}\right) . \mathrm{PBS}$ was employed to assure optimal binding of hEGF to receptors (Nunez et al., 1993). NDLs were composed of SPC and polysorbate 20 in a ratio of 85:15 (w/w), respectively (Dragicevic-Curic et al., 2010). CDLs were made of SPC and stearylamine (weight ratio of 9:1, respectively), and polysorbate 20 . The ratio between SPC and polysorbate 20 was the same as employed for NDLs $(85: 15, \mathrm{w} / \mathrm{w})$. ADLs were composed of SPC and sodium deoxycholate $(85: 15, \mathrm{w} / \mathrm{w})$ (Palac et al., 2014). Table 1 provides an overview of different liposomal compositions. hEGF-containing deformable liposomes were prepared by the dry film method (Ternullo et al., 2017b). SPC and different surfactants, depending on the type of deformable liposomes (Table 1), were dissolved in methanol. The solvent was evaporated on a Büchi Rotavapor R-124 with Büchi Vacuum Pump V-700 (Büchi Labortechnik AG, Flawil, Switzerland) for $1 \mathrm{hr}$ at $55 \mathrm{mbar}$ and $45^{\circ} \mathrm{C}$. The obtained lipid film was then resuspended in 5 $\mathrm{mL}$ of hEGF solution $(20 \mu \mathrm{g} / \mathrm{mL}$ ) in PBS (pH 7.4), previously prepared as described above. Liposomal formulations were stored at $4{ }^{\circ} \mathrm{C}$ for $24 \mathrm{hrs}$ prior further processing. For the cell toxicity and proliferation studies, empty DLs of different surface charges were prepared using the same lipid and surfactant compositions as for hEGF-containing DLs (Table $1)$. 
Table 1. Composition of hEGF-containing deformable liposomes bearing different surface charge.

\subsection{Size reduction of $\mathrm{DLS}$}

Extrusion through polycarbonate membrane (Nuclepore ${ }^{\circledR}$ Track-Etched Membranes, Whatman House, Maidstone, UK) was employed to reduce liposomal size (Ternullo et al., 2017b). The pore size of the polycarbonate membrane and the number of extrusion cycles employed for each liposomal formulation were adjusted to obtain a vesicle ranging between 300-350 $\mathrm{nm}$ in size. Therefore, NDLs were extruded 10 times through $800 \mathrm{~nm}$ pore size membrane and 15 times through $400 \mathrm{~nm}$. CDLs were extruded 10 times through $800 \mathrm{~nm}$ pore size membrane. ADLs were extruded 5 times through $800 \mathrm{~nm}$ pore size membrane. All extruded liposomal formulations were stored at $4{ }^{\circ} \mathrm{C}$ for a minimum of $2 \mathrm{hrs}$ before further usage.

\subsection{Liposomal characterization}

Size distribution and zeta potential analyses of hEGF-containing deformable liposomes were performed using a Malvern Zetasizer Nano - ZS (Malvern, Oxford, UK) (Ingebrigtsen et al., 2017; Ternullo et al., 2017b). For size measurements, all liposomes were diluted 1:50 with filtered PBS ( $0.20 \mu \mathrm{m}$ syringe filter) to achieve an attenuator of 6-7. The zeta potential of all liposomes was determined by diluting each liposomal formulation with filtered $(0.20 \mu \mathrm{m}$ syringe filter) water (1:20 volume ratio). The medium to dilute hEGF-ADLs also contained sodium deoxycholate at the same concentration as in hEGF-ADLs. The zeta potential of free hEGF (hEGF in PBS solution, $\mathrm{pH}$ 7.4) was additionally determined. Prior to the actual measurement, an attenuator of 6-7 was assured for each formulation. Both the size and zeta 
potential measurements were performed in triplicates, at $25^{\circ} \mathrm{C}$ and with an equilibration time of 180 seconds.

\section{5 hEGF content in DLs}

Free (non-encapsulated) hEGF was removed from liposomal dispersions by dialysis (MW cutoff 12,000-14,000 Da, Medicell International Ltd, London, UK). Liposomal dispersion (2 $\mathrm{mL}$ ) was dialysed against $200 \mathrm{~mL}$ of PBS under the sink conditions. Dialysis was carried out for 6 hrs. Both the original and dialysed liposomes were diluted in methanol to dissolve liposomes and hEGF was subsequently quantified using the Enzyme-Linked Immunosorbent Assay (ELISA) as explained in Section 2.6. The entrapment efficiency (\%) was determined by the following relationship $\left(\mu \mathrm{g} \mathrm{hEGF}_{\text {retenate }} / \mu \mathrm{g} \mathrm{hEGF}_{\text {total }}\right) \times 100$, which compares the amount of hEGF in dialysed liposomes and the total amount of hEGF in the original liposomes.

\subsection{Quantification of hEGF}

The quantification of hEGF was performed using ELISA kit for hEGF (Human EGF ELISA

kit, Sigma-Aldrich, Saint Louis, USA), according to the manufacturer's instructions. Aliquots of samples were diluted with ELISA Diluent Buffer B, the solutions added onto an antibodycoated ELISA plate together with standards, and finally incubated for $2.5 \mathrm{hrs}$ at room temperature $\left(23^{\circ} \mathrm{C}\right)$. Diluted biotinylated antibody solution, horseradish peroxidaseconjugated streptavidin solution and tetramethyl benzidine reagent were stepwise added and incubated according to the incubation times recommended in the kit instructions. The final colorimetric reaction was stopped by addition of Stop Solution and absorbance was read immediately at $450 \mathrm{~nm}$ with a SpectraMax 190 Microplate Reader (Molecular Devices, California, USA). A standard curve was obtained using standard hEGF provided in the ELISA 
kit within the concentration range of 1 and $200 \mathrm{pg} / \mathrm{mL}$. Data was fitted with a four-parameter logistic regression equation using SigmaPlot version 13.0 (Systat Software, Inc., San Jose California, USA).

\subsection{In vitro hEGF release}

The in vitro hEGF release from all deformable liposomes was conducted using polyamide membrane (Sartorius polyamide membrane, $0.2 \mu \mathrm{m}$ pore size, Sartorius AG, Gröttingen, Germany) in the Franz diffusion cells $\left(1.77 \mathrm{~cm}^{2}\right.$ diffusion area, $12 \mathrm{~mL}$ acceptor volume; PermeGear, Bethlehem, USA) (Jøraholmen et al., 2017). The acceptor chamber was filled with PBS (pH 7.4) and kept at $32{ }^{\circ} \mathrm{C}$ during the whole experiment using a heating circulator. hEGF-NDLs, hEGF-CDLs and hEGF-ADLs were added in the donor chamber $(600 \mu \mathrm{L})$ and hEGF release from liposomes was investigated for 6 hrs under occlusion. Sampling (500 $\mu \mathrm{L})$ of the acceptor medium was performed after 1, 4 and 6 hrs. Equal volume of fresh PBS was used to replace the withdrawn samples to maintain the sink conditions. hEGF in withdrawn samples was quantified by ELISA as described in Section 2.6. The experiments were performed in duplicates.

\subsection{Preparation of human skin tissue}

The human skin used in this study was obtained from the abdomen of female patients who underwent abdominoplasty and gave their written consent prior the surgery and patient identity was protected. The excess of skin panni from plastic surgery is generally disposed, thus no ethical approval by the Norwegian Ethical Committee was required. The experiments were conducted in accordance with the Declaration of Helsinki Principles. The skin panni obtained from plastic surgery was cleared from the subcutaneous fat and the remaining full 
thickness skin rinsed and moisten with PBS ( $\mathrm{pH} 7.4)$ and stored at $-20{ }^{\circ} \mathrm{C}$. Prior use, skin slices were thawed in PBS at room temperature $\left(23^{\circ} \mathrm{C}\right)$. The thickness of full thickness human skin samples ranged between 1.50 and $1.70 \mathrm{~mm}$.

\subsection{Ex vivo skin penetration studies}

The effect of the different surface charge of deformable liposomes on the skin penetration of hEGF was evaluated using the full thickness human skin, prepared as described above (Section 2.8) (Ternullo et al., 2017b). After thawing, the human skin was cut to slices to closely fit between the donor and acceptor chambers of the same Franz diffusion cells system used for the in vitro drug release experiments (Section 2.7). All liposomal formulations (hEGF-NDLs, hEGF-CDLs and hEGF-ADLs) and control (hEGF in PBS solution) (600 $\mu \mathrm{L})$, respectively were added in the donor chamber and the experiments carried out for 6 hrs under occlusion. Samples $(500 \mu \mathrm{L})$ from the acceptor medium (PBS at $\left.32^{\circ} \mathrm{C}\right)$ were withdrawn after 1, 4 and 6 hrs and replaced with equal volume of fresh PBS, assuring sink conditions. Penetrated (found in the acceptor medium) and non-penetrated (retained in the donor chamber) hEGF were quantified by ELISA following the protocol described in Section 2.6. The experiments were done in triplicates.

\subsection{In vitro cell toxicity and proliferation study}

\subsubsection{Cell culture}

Human foreskin fibroblasts (HF) (CCD-1112Sk, ATCC ${ }^{\circledR}$ CRL-2429 $9^{\mathrm{TM}}$, ATCC, Manassas, USA) were cultured in a $75 \mathrm{~cm}^{2}$ flask with Iscove's modified Dulbecco's medium (IMDM) 
(Sigma-Aldrich Chemie, Steinheim, Germany), supplemented with $10 \%$ (v/v) fetal bovine serum and penicillin-streptomycin at $37{ }^{\circ} \mathrm{C}$ in $5 \% \mathrm{CO}_{2}$.

Human immortalised keratinocytes (HaCaT) (ATCC, Manassas, USA) were cultured on RPMI-1640 medium (Sigma-Aldrich Chemie, Steinheim, Germany) supplemented with $10 \%$ (v/v) fetal bovine serum and penicillin-streptomycin and incubated at $37{ }^{\circ} \mathrm{C}$ in $5 \% \mathrm{CO}_{2}$.

\subsubsection{Cell toxicity and proliferation study}

All liposomal formulations containing hEGF were tested for their toxicity and capability to stimulate cell proliferation in $\mathrm{HF}$ and $\mathrm{HaCaT}$ cells, respectively. To perform the experiment, all liposomes (hEGF-NDLs, hEGF-CDLs and hEGF-ADLs) were prepared to be of a smaller size (ca. $150 \mathrm{~nm}$ ) to avoid sedimentation of liposomal dispersions during the experiments. The amount of entrapped hEGF in the $150 \mathrm{~nm}$-deformable liposomes was quantified as described in Sections 2.5 and 2.6. Moreover, the amount of lipid in all extruded liposomal formulations was determined to confirm that no loss of SPC during the extrusion and separation of unentrapped hEGF from liposomes occurred (data not shown). The cells, previously grown as described in Section 2.10.1, were seeded $(100 \mu \mathrm{L})$ on a 96-wells plate with a density of 25.000 cells $/ \mathrm{mL}$, using the same medium as for cell culture (IMDM and RPMI-1640 for HF and $\mathrm{HaCaT}$, respectively) (Section 2.10.1). The plates were then incubated for $24 \mathrm{hrs}$ at $37^{\circ} \mathrm{C}$ in $5 \% \mathrm{CO}_{2}$ to allow the cells to stabilise and adhere. After $24 \mathrm{hrs}, 10 \mu \mathrm{L}$ of each formulation (hEGF-NDLs, hEGF-CDLs, hEGF-ADLs) were added to the cells and the cells subsequently incubated for 24 and $48 \mathrm{hrs,}$, respectively. Three different lipid concentrations of each liposomal formulation were tested, namely 1,10 and $50 \mu \mathrm{g} / \mathrm{mL}$, which were prepared by diluting each liposomal formulation in the growth medium used for cell culturing. The corresponding concentrations of hEGF in the diluted liposomal dispersions were $0.08,0.8$ and $4 \mu \mathrm{g} / \mathrm{mL}$, respectively. Additionally, the following formulations were tested: 1) PBS, 2) 
empty NDLs, CDLs and ADLs, at the same lipid concentrations as for hEGF-containing liposomes, 3) free hEGF, in PBS in similar concentration as in liposomes. Untreated cells were used as a negative control. After the incubation times, the HF living cells were quantified using CCK-8 kit (Sigma-Aldrich Chemie, Steinheim, Germany). Briefly, $10 \mu \mathrm{L}$ of CCK- 8 were added to each well and incubated for 4 hrs. The absorbance was read at $450 \mathrm{~nm}$ using an Epoch Microplate Spectrophotometer (BioTek Instruments, Vermont, USA). HaCaT living cells were quantified using Cell Proliferation Kit I (MTT) (Roche Diagnostics GmbH, Mannheim, Germany). MTT labeling reagent (10 $\mu \mathrm{L}$, final concentration of $0.5 \mathrm{mg} / \mathrm{mL})$ was added to each well and cells were incubated for 4 hrs under the same conditions. After the incubation period, the formed formazan crystals were dissolved with solubilization buffer $(100 \mu \mathrm{L})$. Absorbance was then measured at $600 \mathrm{~nm}$ (Epoch Microplate Spectrophotometer, BioTek Instruments, Vermont, USA). All experiments were performed in triplicates.

\subsection{Statistical analysis}

All results are expressed as mean \pm standard deviation. To determine statistical significance, Student's $t$-test or one-way ANOVA followed by Bonferroni's multiple comparisons test were performed using GraphPad Prism version 7.00 for Windows (GraphPad Software, La Jolla CA, USA). A $p$ value $<0.05$ was considered significant.

\section{Results and discussion}

In this study, we designed deformable liposomes aiming at improving topical application of hEGF on the skin surface and enhancing the hEGF mitogenic activity. The employment of deformable liposomes may overcome the limitations of hEGF related to topical administration (Zeng and Harris, 2014). To optimize the nanosystem, we particularly focused on the 
liposomal surface charge, which can modulate the liposome's action as skin delivery systems (González-Rodríguez and Rabasco, 2011). This may lead to development of effective liposomal systems exerting an enhancing effect on the hEGF biological activity thus contributing to successful therapeutic outcome of chronic wounds treatment.

\subsection{Characteristics of deformable liposomes}

In the optimisation of effective liposomal formulations destined for dermal drug delivery, it is important to screen liposomal physicochemical properties, not only their vesicle size distribution but also their surface charge (Roberts et al., 2017).

We aimed at obtaining deformable liposomes in a size range of 300-350 $\mathrm{nm}$ which have been reported as able to retain the associated drug in the skin layers while avoiding its absorption into the systemic circulation (du Plessis et al., 1994; Verma et al., 2003). We reported earlier that the extrusion of hydrophilic compound-containing liposomes results in vesicles of homogenous size distributions with low polydispersity index $(\mathrm{PI}=0.06)$ (Ternullo et al., 2017b). Based on those findings, we selected the extrusion as method for liposome size reduction. All hEGF-containing liposomes exhibited vesicle size within the targeted size range with a PI below 0.30 (Table 2). The hEGF-containing CDLs exhibited a slightly larger vesicle size (361.7 nm) and PI (0.30) than NDLs and ADLs (Table 2). Therefore, the incorporation of ionic surfactants in the lipid bilayer resulted in a slight increase in polydispersity as compared to neutral deformable liposomes. Similar findings were reported earlier by Dragicevic-Curic and collaborators (Dragicevic-Curic et al., 2010). Vesicles with a PI of ca. 0.3 can be useful for (trans)dermal delivery, in the case they are unilamellar.

Differently, a large bilayers numbers may affect the vesicle deformability. Therefore, the lamellarity of the liposomes needs to be determined. 
Since we focused on the potential role of the surface charge in the optimization of an effective liposomal formulation for hEGF, the surfactants were selected to prepare DLs with neutral, cationic and anionic surface charge bearing elastic membrane. Particularly, the lipid:polysorbate 20 ratio $(85: 15, \mathrm{w} / \mathrm{w})$ might not be sufficiently high to confer a deformable membrane to the vesicles. However, the choice of using low concentrations of polysorbate 20 was based on previously reported cytotoxicity concerns related to this surfactant (Eskandani et al., 2013). We therefore decided to use a low concentration of surfactant to limit its possible cytotoxic effect in healthy skin cells. Table 2 shows the zeta potentials of the different hEGFcontaining DLs. NDLs exhibited a slightly negative zeta potential $(-0.5 \mathrm{mV})$, which can be considered as neutral. CDLs and ADLs possessed highly positive and negative surface charges (45.8 $\mathrm{mV}$ and $-47.7 \mathrm{mV}$, respectively) due to the presence of stearylamine, known to provide positive zeta potential and sodium deoxycholate, which exerts negative surface charge. The absolute values of the zeta potential of CDLs and ADLs were very similar, thus allowing comparison of the two charged deformable liposomal formulations. The $\mathrm{pH}$ of the medium in which hEGF was dispersed and further used for DLs preparation can affect the protein charge and its polarity (Miklavžin et al., 2018). The zeta potential of hEGF in PBS ( $\mathrm{pH}$ 7.4) was therefore evaluated to determine its surface charge and possibly detect an effect of hEGF on the DLs surface charge. hEGF exhibited a negative zeta potential of - $11.4 \pm 0.5$ $\mathrm{mV}$ when in PBS solution ( $\mathrm{pH}$ 7.4). This can be partially explained by the isoelectric point of hEGF, which is 4.43 (Kim et al., 2015). The higher $\mathrm{pH}$ of the hEGF solution compared to the hEGF isoelectric point determines an increase in the ionized groups in the hEGF molecule thus explaining the negative surface charge of free hEGF. The presence of ionized groups of hEGF in PBS can also indicate that hEGF is mainly incorporated in DLs as a hydrophilic protein (Miklavžin et al., 2018). This suggests that hEGF may accommodate into the aqueous core of DLs to a higher extent, and its negative surface charge can therefore minimally affect 
the DLs surface. This may explain the neutral surface of hEGF-NDLs and the higher absolute values of zeta potential of both hEGF-CDLs and hEGF-ADLs compared to hEGF in solution. On the contrary, hEGF direct binding to the phospholipid bilayers of small unilamellar liposomes has been reported by De La Sierra and co-workers (1992). Therefore, further studies on the hEGF localization within the vesicles need to be performed.

One important factor to consider when developing effective nanosystems is drug entrapment efficiency. A sufficiently high drug incorporation in the nanosystem can assure that the drug would reach the site of action at the therapeutic level thus contributing to successful therapeutic outcome. All liposomes exhibited a high entrapment efficiency (Table 2) with a hEGF content of approx. $16 \mu \mathrm{g} / \mathrm{mL}$. Considering the $\mathrm{EC}_{50}$ of hEGF $(0.08-0.8 \mathrm{ng} / \mathrm{mL})$, the hEGF content in our DLs is promisingly high to assure delivery of the hEGF in therapeutic levels at the targeted skin site. Moreover, the entrapment efficiency appeared not to be affected by liposomal surface charge. Determination of the zeta potential of hEGF in PBS medium ( $\mathrm{pH}$ 7.4) suggested a more hydrophilic nature of hEGF when incorporated in the DLs. Although not yet proven, this finding may indicate that hEGF was predominantly in the aqueous core of the DLs and may explain the similar hEGF content observed for all DLs. The minimal influence of the ionized groups of hEGF on the surface potential of all DLs may indicate limited hEGF interaction with liposomal membrane thus minimally affecting their physicochemical properties, such as zeta potential and entrapment efficiency. The high incorporation of hEGF in all DLs was in accordance with literature. Zhang and co-workers, who incorporated ovalbumin in deformable liposomes made of sodium cholate and stearylamine, reported also similar trend for ionic surfactants. Therefore, the interaction between the two ionic surfactants and lipids in the deformable liposomes can assure a high protein content (Zhang et al., 2017). Moreover, the hEGF content in all liposomes was corresponding to the published data on other phospholipid-based nanosystems, such as solid 
lipid nanoparticles and nanostructured lipid carriers. Gainza and collaborators (2014) prepared vesicles of similar size as our liposomes $(300 \mathrm{~nm})$ and reported a high $\mathrm{hEGF}$ content in both solid lipid and nanostructured lipid carriers (Gainza et al., 2014). Although the difference was not significant, our hEGF-ADLs exhibited slightly higher hEGF content (84.1\%) compared to both NDLs and CDLs ( 80.5 and $80.8 \%$, respectively). Considering the higher hEGF content observed for ADLs, ADLs might be the most suitable nanosystems for hEGF as compared to NDLs and CDLs.

Table 2. Characteristics of the deformable liposomes containing hEGF $(n=3 \pm S D)$.

\subsection{In vitro $h E G F$ release}

To explore the effect of liposomal charge on the hEGF retention on the skin surface, we firstly performed a pilot study on hEGF release from DLs using polyamide membrane in the Franz diffusion cells (Fig.1). No differences in modulating the in vitro hEGF release from the different liposomes were observed. Due to the small population of data $(n=2)$ we could not statistically compare the tested liposomal formulations, however CDLs exhibited a slightly higher hEGF release after 6 hrs than both NDLs and ADLs. Despite the fact that the tested liposomal formulations differ in more than just the surface charge, it may be postulated that the positive surface charge of cationic liposomes may be one of the factors exerting a positive effect on the hEGF release through the artificial membrane. Choi and collaborators have recently found an enhancement in the release of EGF mediated by cationic elastic liposomes as compared to neutral elastic liposomes (Choi et al., 2017). On the other hand, liposomes coated with a negatively charged polymer (xanthan) exhibited a more sustained EGF release (Kaminski et al., 2016); results similar to our ADLs. Polyamide membrane has been earlier employed for testing in vitro release of high molecular weight and hydrophilic drugs, such as 
interferon $\alpha-2 \beta$; the pore size of the membrane $(0.2 \mu \mathrm{m})$ should be sufficiently large to allow the diffusion of the hEGF (Jøraholmen et al., 2017). The choice of targeting relatively large vesicles (ca. $350 \mathrm{~nm}$ ) was also justified considering the pore size membrane; no passage of liposomes through the polyamide membrane occurred as confirmed by the absence of lipids in the acceptor chamber in the Franz cell systems. Earlier, smaller $(200-300 \mathrm{~nm})$ liposomes were partially able to pass through the membrane (Hurler et al., 2012). Therefore, the release of hEGF from all deformable liposomes was mainly attributed to the intrinsic properties of drug retainer. However, polyamide membrane, and other artificial membranes, do not possess the lipid composition and arrangement of the human skin, and this might not correlate well with the in vivo studies (Flaten et al., 2015; Ternullo et al., 2017a). Additionally, polyamide membrane of $200 \mathrm{~nm}$ as pore size is not mimicking the pore size of the skin barrier.

\section{Fig. 1. In vitro hEGF release over 6 hrs from different liposomal formulations through polyamide membrane $(n=2 \pm S D)$.}

\subsection{Ex vivo hEGF penetration through human skin}

The development of effective topical formulations destined to exert local effect onto/into the skin requires testing the drug penetration into/through the skin under the conditions that closely mimic the in vivo conditions (Flaten et al., 2015). The hEGF-containing deformable liposomes were tested under ex vivo conditions using the full thickness human skin and the Franz diffusion cells system. No hEGF was found to penetrate the human skin. This finding indicates that our liposomes are suitable for hEGF retention on the skin surface by reducing any possible systemic absorption of the drug and consequently minimizing undesired side effects. Assuming that hEGF is ionized at the $\mathrm{pH}$ of the medium used to prepare the DLs, 
combined with its characteristic of being a large molecule, the penetration across the full thickness human skin was not expected.

The optimization of hEGF liposomal formulations would require evaluation of hEGF penetration in human skin with compromised skin barrier properties, thus mimicking wounded skin. At this stage of the study, we mainly focused on the effect of the liposomal surface charge on hEGF retention on skin surface. Therefore, the ex vivo skin penetration studies were considered as pilot study to screen the DLs bearing different surface charge. However, one can argue that in the conditions of wounded skin with reduced barrier properties, the findings may differ. The possible penetration through wounded skin needs to be determined.

Moreover, we quantified the amount of hEGF retained onto the human skin surface at the end of the ex vivo skin penetration studies to detect any effect of the liposomal surface charge on skin penetration of hEGF. The amount of hEGF retained onto the skin from PBS solution was of $86.45 \pm 5.40 \%$, whereas NDLs, CDLs and ADLs enabled a hEGF retention of $85.91 \pm 4.38$, $90.17 \pm 5.80$ and $94.39 \pm 2.44 \%$, respectively. We are aware that a low hEGF recovery was observed for both hEGF in solution (control) and hEGF-NDLs and that the hEGF retention onto the skin surface might not be the absolute values for both control and hEGF-NDLs. However, under the same experimental conditions, we could observe a difference in the ability of the DLs bearing different surface charge as drug retainer. The charged deformable liposomes exhibited higher retention of hEGF onto the skin surface compared to both hEGF in solution (control) and NDLs. NDLs showed minimal improvement in skin penetration enhancement of hEGF ( $0.55 \%$ more compared to control). On the other hand, both CDLs and ADLs exhibited higher hEGF retention onto the skin surface, retaining 3.72 and $7.94 \%$ more hEGF onto the skin surface than control, respectively. This enhancement in the depot effect mediated by charged DLs was more evident in comparison to NDLs; the CDLs and ADLs 
retained 4.26 and $8.48 \%$ more hEGF than NDLs, respectively. This indicates that the liposomal surface charge modulated the retention of hEGF on skin surface mediated by DLs. Deformable liposomes have been reported to assure depot of the drug on, in and below the skin barrier (Ascenso et al., 2014). In our case, the comparison of DLs bearing different surface charge allowed us to hypothesise that a better retention of hEGF onto the skin surface is achieved when DLs are negatively charged. This further confirms the ability of anionic nanosystems in assuring a sustained hEGF release over time, as observed in the in vitro study

(Fig. 1). Regarding effective chronic wound therapy, the hEGF depot and its sustained release mediated by ADLs can reduce the frequency of administration thus improving patient compliance, as well as reducing the pain related to dressing change. Pleguezuelos-Villa and co-workers reported similar retention profiles on the skin surface mediated by ADLs. They incorporated naringin, a high molecular weight hydrophilic compound, in deformable liposomes and tested the ex vivo penetration through pig skin. Similarly to what we observed for hEGF-ADLs, more than $90 \%$ of detected compound was found in the donor compartment after the ex vivo penetration study (Pleguezuelos-Villa et al., 2018). The topical hEGF liposomal formulations were applied at hEGF concentration of $16 \mu \mathrm{g} / \mathrm{mL}$, corresponding to the amount of hEGF incorporated in all DLs. The ability of ADLs in retaining almost $95 \%$ of the applied hEGF dose onto the skin within $6 \mathrm{hrs}$ may assure the continuous presence of hEGF at the skin site to induce its receptor and promote the cell proliferation activity. CDLs were slightly superior to ADLs in terms of their ability to enhance hEGF penetration through the skin. The possible explanation may rely on the electrostatic interaction between the positive surface charge of CDLs and the negative charge of the skin surface thus resulting in an increased drug penetration when incorporated in CDLs.

\subsection{Mitogenic effect of hEGF-containing deformable liposomes}


All chronic wounds are characterised by impaired perfusion, oxygenation and limited angiogenesis, and the growth factors have been proposed as an emerging strategy to improve wound healing of chronic wounds by increasing the oxygenation in the wound tissue (Desmet et al., 2018). The incorporation of hEGF in nanosystems has already shown to assure its protection against degradation, a sustained drug release in the administration site and an increase in its therapeutic effect. To prove the effectiveness of our hEGF-containing deformable liposomes, we evaluated their in vitro mitogenic activity in human cells. We chose two dermal cell lines, namely human fibroblasts (HF) and human keratinocytes (HaCaT), whose proliferation in the wound bed would contribute to wound healing (Stunova and Vistejnova, 2018; Werner et al., 2007).

To perform the in vitro cell proliferation assay, we firstly reduced the liposome size to ca. 150 $\mathrm{nm}$ to avoid possible liposome precipitation during the experiment. Therefore, we performed more cycles of extrusion through polycarbonate membrane to obtain the desired liposome size (172.80 $\pm 2.80,191.53 \pm 7.44$ and $168.23 \pm 2.73 \mathrm{~nm}$ for NDLs, CDLs and ADLs, respectively). We also determined the entrapment efficiency, which was found to be of $82.03,79.33$ and $82.52 \%$ for NDLs, CDLs and ADLs, respectively. The additional size reduction did not affect the entrapment efficiency in all liposomes compared to liposomes having a size of $350 \mathrm{~nm}$ (Table 2). The amount of SPC in all liposomes was also determined in order to assure any SPC loss during the extrusion and separation of unentrapped hEGF by dialysis. A SPC loss below $3.5 \%$ was found for all liposomal formulations (data not shown).

Regarding hEGF-NDLs, a mitogenic effect in HF was observed only at the highest lipid concentration $(50 \mu \mathrm{g} / \mathrm{mL})$ (Fig. 2a). This effect was already evident after 24 hrs treatment, when a significant increase $(p<0.03)$ was observed compared to the untreated HF cells (control) and became even more evident after 48 hrs treatment $(p<0.0001)$. A dosedependency was not observed after $24 \mathrm{hrs}$ treatment, whereas it was evident after $48 \mathrm{hrs}$ 
treatment. This suggests that a shorter incubation time may not be sufficient to detect the hEGF concentration effect in HF. The mitogenic activity of hEGF-NDLs was, at the highest lipid concentration, stronger than activity of free hEGF (hEGF in PBS; $p<0.03$ ) (Fig. 2a, d) after $48 \mathrm{hrs}$ treatment.

hEGF-CDLs at both 1 and $10 \mu \mathrm{g} / \mathrm{mL}$ lipid concentrations showed no significant improvement on HF proliferation after $24 \mathrm{hrs}$ treatment compared to control (Fig. 2b). However, the highest concentration of hEGF-CDLs exhibited a lower cell proliferation rate compared to the other tested formulations (Fig. 2b, d). This dose-dependent cytotoxic effect might be related to the presence of stearylamine, which can interact with the cell membrane thus damaging membranes' integrity and function (Tahara et al., 2018). However, empty CDLs at the same (highest) lipid concentration were not toxic to HF, therefore, the low cell proliferation observed for the highest concentration of hEGF-CDLs might be contributed to the high dose of hEGF ( $4 \mu \mathrm{g} / \mathrm{mL})$, rather than the lipid. Interestingly, after $48 \mathrm{hrs}$ treatment, hEGF-CDLs at lipid concentration of $10 \mu \mathrm{g} / \mathrm{mL}$ increased the HF proliferation by $40 \%$ as compared to control $(p<0.0001)$ (Fig. 2b). This indicates that up to $10 \mu \mathrm{g} / \mathrm{mL}$ lipid concentration and 0.8 $\mu \mathrm{g} / \mathrm{mL}$ hEGF concentration, hEGF-CDLs are not toxic to cells and can be employed for wound healing treatment. This positive effect was not detectable after $24 \mathrm{hrs}$ treatment indicating that the cell response is dependent on the incubation time, as previously reported (Bertoncelj et al., 2014). The highest tested concentration of hEGF-CDLs showed lower mitogenic effect after $48 \mathrm{hrs}$ treatment thus confirming the dose-dependency of cellular response.

hEGF-ADLs did not exhibit any significant enhancement in terms of HF proliferation after 24 hrs treatment compared to control and the other tested formulations (Fig. 2c, d). However, after $48 \mathrm{hrs}$, hEGF-ADLs at both lipid concentrations (10 and $50 \mu \mathrm{g} / \mathrm{mL})$ exhibited a greater mitogenic effect than untreated cells $(p<0.002)$, allowing 48 and $77 \%$ HF more proliferation 
than control, respectively. Additionally, a hEGF concentration-dependency effect was evident after $48 \mathrm{hrs}$ treatment, as observed for hEGF-NDLs. The highest lipid and hEGF concentrations in hEGF-ADLs enhanced HF proliferation to a greater extent than both empty ADLs and free hEGF, by 33 and $45 \%$ respectively (Fig. 2c, d). This further confirms the findings for hEGF-NDLs and hEGF-CDLs, indicating that the keratinocytes response depends both on the incubation time and hEGF/lipid concentrations. This is in accordance with previously published data on the keratinocytes response when exposed to growth factorscontaining nanosystems (Bertoncelj et al., 2014). Comparing the three hEGF-containing liposomal formulations, the hEGF-ADLs assured the greatest mitogenic effect when tested at the highest lipid and hEGF concentrations (Fig. 2). Ex vivo skin penetration studies revealed the depot effect of hEGF-ADLs, thus enabling its sustained release into human skin. This can assure a continuous binding of hEGF to its receptor, thus constantly activating the signalling pathway and prolonging the proliferation effect in cells. The similar trend was observed for hEGF incorporated in other nanosystems, such as solid lipid nanoparticles and nanostructured lipid carriers (Gainza et al., 2014).

The mitogenic effect of the hEGF liposomal formulations on HaCaT cells is shown in Fig. 3. The 24 hrs treatment showed that only the highest tested hEGF concentration $(4 \mu \mathrm{g} / \mathrm{mL})$, in both NDLs and ADLs was more effective in enhancing cell proliferation compared to both untreated cells and free hEGF (Fig. 3a, c, d). hEGF-NDLs assured 26 and $32 \%$ more cell proliferation more than control and free hEGF, respectively. On the other hand, hEGF-ADLs increased the percentage of living cells by respectively 36 and $41 \%$ more compared to control and free hEGF. Therefore, between the two liposomal formulations, anionic deformable liposomes (hEGF-ADLs) exhibited stronger mitogenic activity compared to hEGF-NDLs. This confirms the findings from the cell proliferation studies in HF, which, taken together, highlights the superiority of ADLs in improving the mitogenic effect of hEGF as compared to 
NDLs and CDLs. Regarding CDLs, 24 hrs treatment revealed the same trend as observed for the HF cell line (Fig. 2b). Up to a lipid concentration of $10 \mu \mathrm{g} / \mathrm{mL}$, hEGF-containing CDLs showed to be not toxic also in $\mathrm{HaCaT}$ cell line, while a lower cell proliferation rate was observed for hEGF-CDLs at the highest lipid concentration (Fig. 3b). HaCaT cells treated for $48 \mathrm{hrs}$ with all liposomal formulations proliferated to a lower extent compared to control (Fig. 3a, b, c). These findings differ from the results obtained with the HF cell line (Fig. 2a, b, c), showing that the growth rate is also dependent on the cell line employed for the in vitro cell proliferation assay in agreement with Gainza et al. (2014). In the case of hEGF-CDLs at the highest lipid and hEGF concentrations, lower mitogenic effect was also observed for both empty CDLs and free hEGF (Fig. 3b, d). Considering NDLs and CDLs, the lower proliferation activity might be related to the presence of polysorbate 20 . Contri and coworkers observed the same decrease in cellular viability when nanocapsules containing similar surfactant, polysorbate 80, were tested on keratinocytes (Contri et al., 2016). The stronger cytotoxic effect observed for hEGF-CDLs compared to hEGF-NDLs might be due to a synergistic effect between polysorbate 20 and stearylamine. The stronger toxicity observed for hEGF-CDLs compared to empty CDLs highlights the dose-dependency response of HaCaT to hEGF, as found for HF cell line (Fig. 2b). In addition to the influence of the surfactants, the reduced mitogenic effect observed at the highest concentration of hEGF-CDLs in both cell lines might be explained by the results from in vitro drug release (Fig. 1) and ex vivo skin penetration studies. CDLs were able to enhance both in vitro hEGF release and its skin penetration through the SC, resulting in a faster hEGF release from the cationic vesicles compared to neutral and anionic ones. This might cause a faster uptake of hEGF followed by a rapid clearance from the site of action, thus reducing its therapeutic effect. ADLs enabled a more sustained hEGF release and depot onto the skin surface, which could explain the prolonged and stronger mitogenic activity of hEGF when 
incorporated in ADLs. This might be considered a further advantage in terms of reduction of administration frequency. Moreover, the enhanced long-term mitogenic effect of hEGF when incorporated in ADLs compared to the free hEGF suggests that ADLs offer protection of the drug, assuring longer stability and thus contributing to an enhanced therapeutic effect.

Fig. 2. Mitogenic effect of hEGF-containing deformable liposomes on human skin fibroblasts (HF) $(n=3 \pm$ SD).

Fig. 3. Mitogenic effect of hEGF-containing deformable liposomes on human immortalized keratinocytes (HaCaT) $(n=3 \pm S D)$.

We confirmed the potential of anionic deformable liposomes in in vitro conditions; however, their real clinical potential will have to be confirmed in animal and clinical studies.

\section{Conclusions}

Neutral, cationic and anionic deformable liposomes containing hEGF were prepared to improve the mitogenic activity of growth factors when topically applied on the skin surface. The formulations were evaluated in terms of their physicochemical characteristics, in vitro drug release, ex vivo drug penetration through human skin, as well as the in vitro mitogenic activity in human fibroblasts and keratinocytes. All deformable liposomes exhibited high hEGF content, with a slightly higher load for anionic deformable liposomes. hEGF-containing anionic deformable liposomes exhibited the most sustained hEGF in vitro release and assured a depot on the ex vivo human skin as compared to neutral and cationic deformable liposomes. 
In addition, anionic deformable liposomes revealed to be the most effective in enhancing the mitogenic effect of hEGF in $\mathrm{HF}$ and $\mathrm{HaCaT}$ cell lines compared to the neutral and cationic deformable liposomes as well as hEGF in solution. Anionic deformable liposomes have shown to be a promising system for improved chronic wound therapy. Their clinical potential will have to be further explored.

\section{Conflict of interest}

The authors declare no conflicts of interest.

\section{Acknowledgements}

The authors thank Lipoid GmbH for kindly providing the lipids used in the experiments.

\section{REFERENCES}

Alemdaroğlu, C., Degim, Z., Celebi, N., Şengezer, M., Alömeroglu, M., Nacar, A., 2008. Investigation of epidermal growth factor containing liposome formulation effects on burn wound healing. J. Biomed. Mater. Res. A 85, 271-283.

Ascenso, A., Salgado, A., Euletério, C., Garcia Praça, F., Lopes Badra Bentley, M.V., Marques, H.C., Oliveira, H., Santos, C., Simões, S., 2014. In vitro and in vivo topical delivery studies of tretinoin-loaded ultradeformable vesicles. Eur. J. Pharm. Biopharm. 88, 48-55. 
Ashtikar, M., Wacker, M.G., 2018. Nanopharmaceuticals for wound healing - Lost in translation? Adv. Drug Deliv. Rev. https://doi.org/10.1016/j.addr.2018.03.005

Bertoncelj, V., Pelipenko, J., Kristl, J., Jeras, M., Cukjati, M., Kocbek, P., 2014. Development and bioevaluation of nanofibers with blood-derived growth factors for dermal wound healing. Eur. J. Pharm. Biopham. 88, 64-74.

Boateng, J., Catanzano, O., 2015. Advanced therapeutic dressings for effective wound healing - A review. J. Pharm. Sci. 104, 3653-3680.

Cevc, G., 2003. Transdermal drug delivery of insulin with ultradeformable carriers. Clin. Pharmacokinet. 42, 461-474.

Cevc, 2004. Lipid vesicles and other colloids as drug carriers on the skin. Adv. Drug Deliv. Rev. 56, 675-711.

Choi, J.U., Lee, S.W., Pangeni, R., Byun, Y., Yoon, I-S., Park, J.W., 2017. Preparation and in vivo evaluation of cationic elastic liposomes comprising highly skin-permeable growth factors combined with hyaluronic acid for enhanced diabetic wound-healing therapy. Acta Biomater. $57,197-215$.

Contri, R.V., Fiel, L.A., Alnasif, N., Pohlmann, A.R., Guterres, S.S., Schäfer-Korting, M., 2016. Skin penetration and dermal tolerability of acrylic nanocapsules: Influence of the surface charge and a chitosan gel used as vehicle. Int. J. Pharm. 507, 12-20.

De La Sierra, I.M.L., Vincent, M., Padron, G., Gallay, J., 1992. Interaction of recombinant human epidermal growth factor with phospholipid vesicles. A steady-state and time-resolved fluorescence study of the bis-tryptophan sequence (TRP 49 -TRP $\left.{ }_{50}\right)$. Eur. Biophys. J. 21, 337344. 
Desmet, C.M., Préat, V., Gallez, B., 2018. Nanomedicine and gene therapy for the delivery of growth factors to improve perfusion and oxygenation in wound healing. Ad. Drug Deliv. Rev. Doi: 10.1016/j.addr.2018.02.001.

Dragicevic-Curic, N., Gräfe, S., Gitter, B., Winter, S., Fahr, A., 2010. Surface charged temoporfin-loaded flexible vesicles: In vitro skin penetration studies and stability. Int. J. Pharm. 384, 100-108.

du Plessis, J., Ramachandran, C., Weiner, N., Müller, D.G., 1994. The influence of particle size of liposomes on the deposition of drug into the skin. Int. J. Pharm. 103, 277-282.

Eskandani, M., Hamishehkar, H., Ezzati Nazhad Dolatabadi, J., 2013. Cyto/Genotoxicity study of polyoxyethylene (20) sorbitan monolaurate (tween 20). DNA Cell Biol. 32, 498-503.

Flaten, G.E., Palac, Z., Engesland, A., Filipović-Grčić, J., Vanić, Ž., Škalko-Basnet, N., 2015. In vitro skin models as a tool in optimization of drug formulation. Eur. J. Pharm. Sci. 75, 1024.

Gainza, G., Pastor, M., Aguirre, J.J., Villullas, S., Pedraz, J.L., Hernandez, R.M., Igartua, M., 2014. A novel strategy for the treatment of chronic wounds based on the topical administration of rhEGF-loaded lipid nanoparticles: In vitro bioactivity and in vivo effectiveness in healing-impaired $d b / d b$ mice. J. Control. Release 185, 51-61.

Gainza, G., Villullas, S., Pedraz, J.L., Hernandez, R.M., Igartua, M., 2015b. Advances in drug delivery systems (DDSs) to release growth factors for wound healing and skin regeneration. Nanomedicine: NBM 11, 1551-1573.

Garcia-Orue, I., Gainza, G., Gutierrez, F.B., Aguirre, J.J., Evora, C., Pedraz, J.L., Hernandez, R.M., Delgado, A., Igartua, M., 2017a. Novel nanofibrous dressings containing rhEGF and Aloe vera for wound healing applications. Int. J. Pharm. 523, 556-566. 
Garcia-Orue, I., Pedraz, J.L., Hernandez, R.M., Igartua, M., 2017b. Nanotechnology-based delivery systems to release growth factors and other endogenous molecules for chronic wound healing. J. Drug Deliv. Sci. Technol. 42, 2-17.

González-Rodríguez, M.L., Rabasco, A.M., 2011. Charged liposomes as carrier to enhance the permeation through the skin. Expert Opin. Drug Deliv. 8, 857-871.

Hamdan, S., Pastar, I., Drakulich, S., Dikici, E., Tomic-Canic, M., Deo, S., Daunert, S., 2017. Nanotechnology-driven therapeutic interventions in wound healing: potential uses and applications. ACS Cent. Sci. 3, 163-175.

Hurler, J., Berg, O.A., Skar, M., Conradi, A.H., Johnsen, P.J., Škalko-Basnet, N., 2012. Improved burns therapy: Liposomes-in-hydrogel delivery system for mupirocin. J. Pharm. Sci. 101, 3906-3915.

Ingebrigtsen, S.G., Škalko-Basnet, N., de Albuquerque Cavalcanti Jacobsen, C., Holsæter, A.M., 2017. Successful co-encapsulation of benzoyl peroxide and chloramphenicol in liposomes by a novel manufacturing method - dual asymmetric centrifugation. Eur. J. Pharm. Sci. 97, 192-199.

Jahromi, M.A.M., Zangabad, P.S., Basri, S.M.M., Zangabad, K.S., Ghamarypour, A., Aref, A.R., Karimi, M., Hamblin, M.R., 2018. Nanomedicine and advanced technologies for burns: preventing infection and facilitating wound healing. Adv. Drug Deliv. Rev. 123, 33-64.

Jain, S., Patel, N., Shah, M.K., Khatri, P., Vora, N., 2017. Recent advances in lipid-based vesicles and particulate carriers for topical and transdermal application. J. Pharm. Sci. 106, 423-445.

Jeon, S.O., Hwang, H.J., Oh, D.H., Seo, J.E., Chun, K.H., Hong, S.M., Kim, M.J., Kim, W.C., Park, M.S., Yoon, C.H., Min, K.H., Suh, C.W., Lee, S., 2012. Enhanced percutaneous 
delivery of recombinant human epidermal growth factor employing nano-liposome system. J. Microencapsul. 29, 234-241.

Jøraholmen, M.W., Basnet, P., Acharya, G., Škalko-Basnet, N., 2017. PEGylated liposomes for topical vaginal therapy improve delivery of interferon alpha. Eur. J. Pharm. Biopharm. $113,132-139$.

Kalashnikova, I., Das, S., Seal, S., 2015. Nanomaterials for wound healing: scope and advancement. Nanomedicine (Lond.) 10, 2593-2612.

Kaminski, G.A.T., Sierakowski, M.R., Pontarolo, R., dos Santos, L.A., de Freitas, R.A., 2016. Layer-by-layer polysaccharide-coated liposomes for sustained delivery of epidermal growth factor. Carbohydr. Polym. 140, 129-135.

Kim, N.A., Lim, D.G., Lim, J.Y., Kim, K.H., Jeong, S.H., 2015. Fundamental analysis of recombinant human epidermal growth factor in solution with biophysical methods. Drug Dev. Ind. Pharm. 41, 300-306.

Miklavžin, A., Cegnar, M., Kerč, J., Kristl, J., 2018. Effect of surface hydrophobicity of therapeutic protein loaded in polyelectrolyte nanoparticles on transepithelial permeability. Acta Pharm. 68, 275-293.

Mota, A.H., Rijo, P., Molpeceres, J., Pinto Reis, C., 2017. Broad overview of engineering of functional nanosystems for skin delivery. Int. J. Pharm. 532, 710-728.

Nunez, M., Mayo, K.H., Starbuck, C., Lauffenburger, D., 1993. pH sensitivity of epidermal growth factor receptor complexes. J. Cell. Biochem. 51, 312-321.

Palac, Z., Engesland, A., Flaten, G.E., Škalko-Basnet, N., Filipović-Grčić, J., Vanić, Ž., 2014. Liposomes for (trans)dermal drug delivery: the skin-PVPA as a novel in vitro stratum corneum model in formulation development, J. Liposome Res. 24, 313-322. 
Paul, A., Cevc, G., Bachhawat, B.K., 1995. Transdermal immunization with large proteins by means of ultradeformable drug carriers. Eur. J. Immunol. 25, 3521-3524.

Paul, A., Cevc, G., Bachhawat, B.K., 1998. Transdermal immunisation with an integral membrane component, gap junction protein, by means of ultradeformable drug carriers, transfersomes. Vaccine 16, 188-195.

Pleguezuelos-Villa, M., Mir-Palomo, S., Díez-Sales, O., Vila Buso, M.A.O., Ruiz Sauri, A., Nácher, A., 2018. A novel ultradeformable liposomes of Naringin for anti-inflammatory therapy. Colloids Surf. B. Biointerfaces 162, 265-270.

Roberts, M.S., Mohammed, Y., Pastore, M.N., Namjoshi, S., Yousef, S., Alinaghi, A., Haridass, I.N., Abd, E., Leite-Silva, V.R., Benson, H.A.E., Grice, J.E., 2017. Topical and cutaneous delivery using nanosystems. J. Control. Release 247, 86-105.

Saghazadeh, S., Rinoldi, C., Schot, M., Kashaf, S.S., Sharifi, F., Jalilian, E., Nuutila, K., Giatsidis, G., Mostafalu, P., Derakhshandeh, H., Yue, K., Swieszkowski, W., Memic, A., Tamayol, A., Khademhosseini, A., 2018. Drug delivery systems and materials for wound healing applications. Adv. Drug Deliv. Rev. https://doi.org/10.1016/j.addr.2018.04.008

Sala, M., Diab, R., Elaissari, A., Fessi, H., 2018. Lipid nanocarriers as skin drug delivery systems: Properties, mechanisms of skin interactions and medical applications. Int. J. Pharm. $535,1-17$.

Stunova, A., Vistejnova, L., 2018. Dermal fibroblasts - A heterogenous population with regulatory function in wound healing. Cytokine Growth Factor Rev. 39, 137-150.

Tahara, K., Kobayashi, M., Yoshida, S., Onodera, R., Inoue, N., Takeuchi, H., 2018. Effects of cationic liposomes with stearylamine against virus infection. Int. J. Pharm. 543, 311-317. 
Ternullo, S., de Weerd, L., Flaten, G.E., Holsæter, A.M., Škalko-Basnet, N., 2017a. The isolated perfused human skin flap model: A missing link in skin penetration studies? Eur. J. Pharm. Sci. 96, 334-341.

Ternullo, S., de Weerd, L., Holsæter, A.M., Flaten, G.E., Škalko-Basnet, N., 2017b. Going skin deep: A direct comparison of penetration potential of lipid-based nanovesicles on the isolated perfused human skin flap model. Eur. J. Pharm. Biopharm. 121, 14-23.

Verma, D.D., Verma, S., Blume, G., Fahr, A., 2003. Particle size of liposomes influences dermal delivery of substances into skin. Int. J. Pharm. 258, 141-51.

Werner, S., Krieg, T., Smola, H., 2007. Keratinocyte-fibroblast interactions in wound healing. J. Investig. Dermatol. 127, 998-1008.

Xue, M., Zhao, R., Lin, H., Jackson, C., 2018. Delivery systems of current biologicals for the treatment of chronic cutaneous wounds and severe burns. Adv. Drug Deliv. Rev. https://doi.org/10.1016/j.addr.2018.03.002

Zeng, F., Harris, R.C., 2014. Epidermal growth factor, from gene organization to bedside. Semin. Cell. Dev. Biol. 28, 2-11.

Zhang, Y., Ng, W., Feng, X., Cao, F., Xu, H., 2017. Lipid vesicular nanocarrier: Quick encapsulation efficiency determination and transcutaneous application. Int. J. Pharm. 516, 225-230. 
Figures and figure legends:

Fig. 1. In vitro hEGF release over 6 hrs from different liposomal formulations through polyamide membrane $(n=2 \pm S D)$.

Fig. 2. Mitogenic effect of hEGF-containing deformable liposomes on human skin fibroblasts (HF) $(n=3 \pm S D)$.

The mitogenic effect of hEGF-NDLs (a), hEGF-CDLs (b) and hEGF-ADLs (c) on HF cells was investigated for 24 and 48 hours, respectively. NDLs, CDLs and ADLs represent empty neutral, cationic and anionic deformable liposomes, respectively. Untreated cells are referred as control. The d represents the mitogenic effect of hEGF in PBS (free hEGF) at same tested concentrations as for hEGF entrapped in liposomes.

${ }^{\mathrm{a}}$ Significantly higher than control $\left({ }^{\mathrm{a}} p<0.03,{ }^{\mathrm{a}} p<0.002,{ }^{\mathrm{a}} \mathrm{p} p<0.0001\right)$

Fig. 3. Mitogenic effect of hEGF-containing deformable liposomes on human immortalized keratinocytes $(\mathrm{HaCaT})(\mathrm{n}=3 \pm \mathrm{SD})$.

The mitogenic effect of hEGF-NDLs (a), hEGF-CDLs (b) and hEGF-ADLs (c) on HaCaT cells was investigated for 24 and 48 hours, respectively. NDLs, CDLs and ADLs represent empty neutral, cationic and anionic deformable liposomes, respectively. Untreated cells are referred as control. The d represents the mitogenic effect of drug in PBS (free hEGF) at same tested concentrations as for hEGF entrapped in liposomes.

${ }^{\mathrm{a}}$ Significantly higher than control $\left({ }^{\mathrm{a}} p<0.03,{ }^{\mathrm{a}} p<0.002,{ }^{\mathrm{a}} \mathrm{p} p<0.0001\right)$ 
Tables and table legends:

Table 1. Composition of hEGF-containing deformable liposomes bearing different surface charge.

${ }^{\mathrm{a}}$ All liposomal formulations were prepared to a final volume of $5 \mathrm{~mL}$.

${ }^{\mathrm{b}}$ Empty NDLs, CDLs and ADLs (without hEGF) were prepared using the same composition as for hEGF-NDLs, hEGF-CDLs and hEGF-ADLs, respectively.

Table 2. Characteristics of the deformable liposomes containing hEGF $(n=3 \pm S D)$.

aPolydispersity index 
hEGF

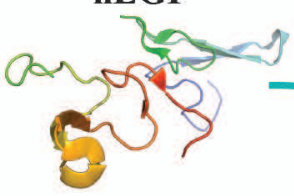

Cationic deformable liposome

(CDL) $\oplus$

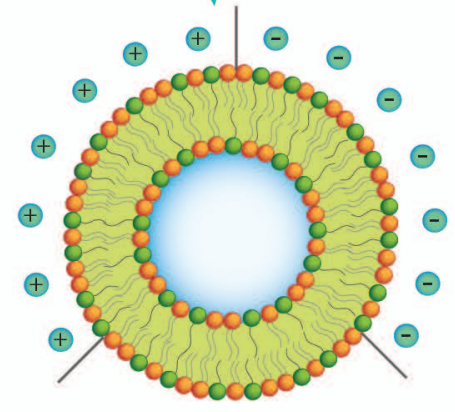

Neutral deformable liposome (NDL)
Anionic deformable liposome (ADL)

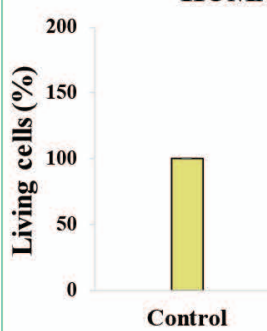

\section{$48 \mathrm{~h}$ treatment}

$\square 1 \mu \mathrm{g} / \mathrm{mL} \quad \square 10 \mu \mathrm{g} / \mathrm{mL} \quad \square 50 \mu \mathrm{g} / \mathrm{mL}$
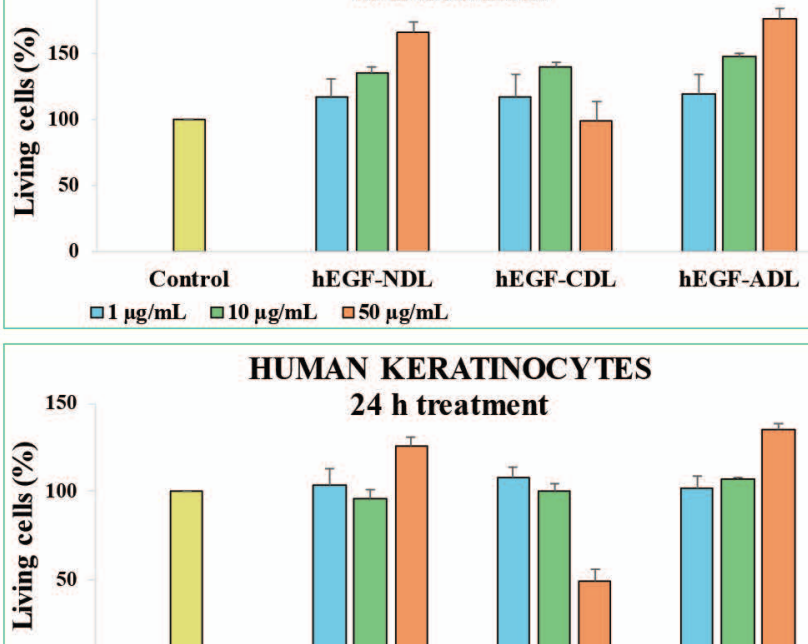

HUMAN KERATINOCYTES

$150 \quad 24 \mathrm{~h}$ treatment
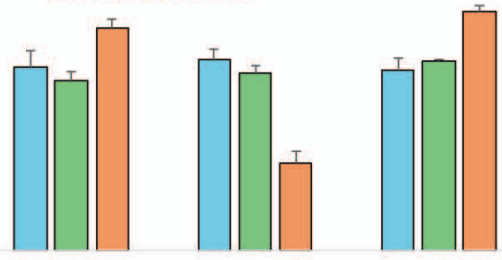

Control

hEGF-NDL

hEGF-CDI

hEGF-ADL 


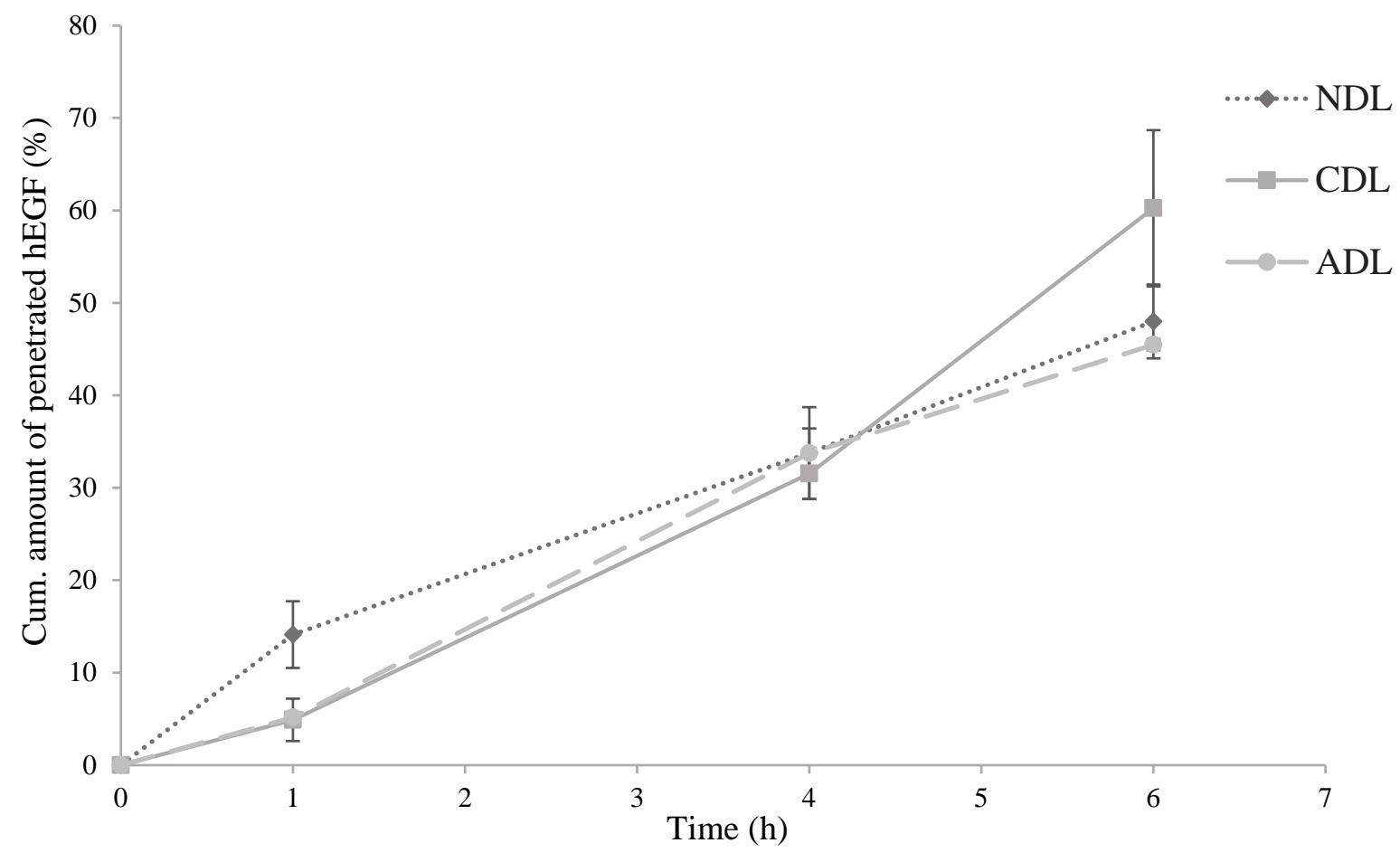


Table 1. Composition of hEGF-containing deformable liposomes bearing different surface charge.

$\begin{array}{cccccc}\text { Liposomes }^{\mathrm{a}, \mathrm{b}} & \text { hEGF } & \text { SPC } & \text { Polysorbate 20 } & \text { Stearylamine } & \text { Sodium deoxycholate } \\ (\mu \mathrm{g}) & (\mathrm{mg}) & (\mathrm{mg}) & (\mathrm{mg}) & (\mathrm{mg})\end{array}$

\begin{tabular}{lccccc}
\hline hEGF-NDLs & 100 & 170 & 30 & - & - \\
hEGF-CDLs & 100 & 153 & 30 & 17 & - \\
hEGF-ADLs & 100 & 170 & - & - & 30
\end{tabular}

\footnotetext{
${ }^{\mathrm{a}}$ All liposomal formulations were prepared to a final volume of $5 \mathrm{~mL}$.

${ }^{\text {b}}$ Empty NDLs, CDLs and ADLs (without hEGF) were prepared using the same composition as for hEGF-NDLs, hEGF-CDLs and hEGF-ADLs, respectively.
} 
Table 2. Characteristics of the deformable liposomes containing hEGF ( $n=3 \pm$ SD).

\begin{tabular}{lcccc} 
Formulation & $\begin{array}{c}\text { Size } \\
(\mathrm{nm})\end{array}$ & $\mathrm{PI}^{\mathrm{a}}$ & $\begin{array}{c}\text { Zeta potential } \\
(\mathrm{mV})\end{array}$ & $\begin{array}{c}\text { Entrapment efficiency } \\
(\%)\end{array}$ \\
\hline hEGF-NDLs & $350.0 \pm 8.2$ & $0.22 \pm 0.01$ & $-0.5 \pm 0.6$ & $80.5 \pm 1.5$ \\
hEGF-CDLs & $361.7 \pm 32.5$ & $0.30 \pm 0.02$ & $45.8 \pm 1.8$ & $80.8 \pm 0.7$ \\
hEGF-ADLs & $322.7 \pm 12.5$ & $0.26 \pm 0.00$ & $-47.7 \pm 5.8$ & $84.1 \pm 6.1$ \\
\hline
\end{tabular}

${ }^{\text {apolydispersity index }}$ 